\title{
Central Nervous System Complications due to Human Herpesvirus 6 Infection in Pediatric Age Group
}

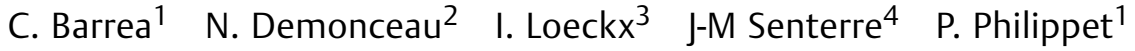 \\ ${ }^{1}$ Department of Paediatrics, Centre Hospitalier Chrétien, CHC, \\ Liège, Belgium \\ 2 Department of Neuropédiatrics, Centre Hospitalier Chrétien, CHC, \\ Liège, Belgium \\ 3 Pediatric Intensive Care Unit, Centre Hospitalier Chrétien, CHC, \\ Liège, Belgium \\ ${ }^{4}$ Master in Paediatrics, University of Liège, ULg, Belgium \\ J Pediatr Neurol \\ Address for correspondence Barrea Christophe, MD, Centre \\ Hospitalier Chrétien, 447-449 Rue St Nicolas, 4420 Montegnée, \\ Belgium (e-mail: chbarrea@gmail.com).
}

\author{
Abstract \\ Keywords \\ - brain \\ - HHV-6 infection \\ - encephalitis \\ - pediatric \\ - febrile seizure
}

Human herpesvirus 6 (HHV-6) has been demonstrated to be a neurotropic virus and has been linked to several neurological complications associated with a wide range of clinical outcomes. Here, we present data from immunocompetent children with a central neurological condition and intrathecal DNA detection of HHV-6 virus. Through these cases, physiopathology, investigations, and outcome of the disease will be described. Finally, specific treatment will be discussed.

\section{Introduction}

Human herpesvirus 6 (HHV-6) is one of the most prevalent herpesviruses in humans. Two distinct variants have been described, HHV-6A and HHV-6B. HHV-6A is commonly encountered in central Africa, and no disease, neurological or otherwise, has been causally linked to it. In contrast, primary infection with HHV-6B is universal, predominantly occurs in early childhood and manifests as a self-limiting febrile illness with or without an exanthema subitum rash. ${ }^{1}$ The majority of the population is HHV-6 seropositive by the age of 3 years, with a greater incidence between 6 and 18 months. The virus is also well established as a cause of neurological disease. ${ }^{2}$

The HHV-6A and HHV-6B envelope glycoprotein complex binds respectively to human CD46 and CD134 expressed on almost all cell types, especially on monocytes-macrophages. HHV-6 can integrate their genomes into the host telomeres of host chromosomes in proximity to the subtelomeric region. $^{2}$ Congenital transmission ${ }^{3}$ and contamination with stem cell transplantation ${ }^{4}$ are also possible, with human HHV-6 DNA persistence afterwards. The integrated virus can remain silent or can reactivate, indicating that integration

received

January 11, 2019

accepted after revision

February 8, 2019 represents a mechanism by which these viruses might achieve latency. HHV-6 reactivation may be favored by depletion of CD4+ cells and therefore, affects mainly immunocompromised patients; however, serious infections in immunocompetent patients have been described.

HHV-6 infection may be accompanied by central nervous system (CNS) disease. Here, we report four pediatric cases to highlight the wide spectrum of clinical presentation, medical care, and outcomes.

\section{Patients and Methods}

\section{Patients}

Children under 18 years were drawn from a retrospective study on central neurologic infection, performed in the Centre Hospitalier Chrétient (CHC) Esperance in Liege, during the year 2015.

\section{Methods}

A quantitative human HHV-6 polymerase chain reaction (PCR) was performed on the cerebrospinal fluid (CSF) samples of 10 children who present fever and central neurological manifestations. Among them, four patients had no

Copyright $\odot$ by Georg Thieme Verlag KG, DOI https://doi.org/ Stuttgart · New York 10.1055/s-0039-1683440. ISSN $1304-2580$. 
HHV-6 Infection of the CNS Barrea et al.

Table 1 Laboratory findings of cases reported

\begin{tabular}{|c|c|c|c|c|c|c|c|c|c|c|}
\hline \multirow[t]{3}{*}{ Case } & \multicolumn{4}{|c|}{ Blood test } & \multicolumn{5}{|c|}{ Cerebrospinal fluid } & \multirow[t]{3}{*}{ Cultures and other PCR } \\
\hline & \multirow{2}{*}{$\begin{array}{c}\mathrm{WBC} \\
\left(10^{3} \mid\right. \\
\left.\mathrm{mm}^{3}\right)\end{array}$} & \multirow{2}{*}{$\begin{array}{l}\mathrm{CRP} \\
\text { (mg/L) }\end{array}$} & \multicolumn{2}{|c|}{ HHV-6 } & \multirow{2}{*}{$\begin{array}{l}\text { WBC } \\
\left(/ \mathrm{mm}^{3}\right)\end{array}$} & \multirow{2}{*}{$\begin{array}{l}\mathrm{RBC} \\
\left(/ \mathrm{mm}^{3}\right)\end{array}$} & \multirow{2}{*}{$\begin{array}{l}\text { Glu } \\
\text { (mg/dL) }\end{array}$} & \multirow{2}{*}{$\begin{array}{l}\text { Protein } \\
\text { level } \\
\text { (mg/L) }\end{array}$} & \multirow{2}{*}{$\begin{array}{l}\text { HHV-6 } \\
\text { (copyl } \\
\mathrm{mL} \text { ) }\end{array}$} & \\
\hline & & & $\lg M$ & IgG & & & & & & \\
\hline 1 & 15 & 200 & + & - & 73 & 3 & 83.4 & 560 & 3,660 & \multirow{4}{*}{$\begin{array}{l}\text { There was no other infectious } \\
\text { agent detected in cultures, or by } \\
\text { multiplex PCR (Biofire) excluding } \\
\text { the presence of Escherichia coli, } \\
\text { Haemophilus influenzae, Listeria } \\
\text { monocytogenes, Neisseria } \\
\text { meningitides, Streptococcus } \\
\text { agalactiae, Streptococcus } \\
\text { pneumoniae, Cryptococcus } \\
\text { neoformans, and gatti, } \\
\text { cytomegalovirus, enterovirus, } \\
\text { herpes simplex } 1 \text { and 2, human } \\
\text { parechovirus, and varicella } \\
\text { zoster virus in the CSF }\end{array}$} \\
\hline 2 & 17 & 9.6 & 1 & 1 & 59 & 13 & 69.5 & 406 & 2,770 & \\
\hline 3 & 2 & 38.4 & 1 & 1 & 1 & 51 & 53.4 & 668 & 2,390 & \\
\hline 4 & 16 & 6.4 & 1 & 1 & 2 & 1 & 85.1 & 142 & 1,480 & \\
\hline
\end{tabular}

Abbreviations: CRP, C-reactive protein; CSF, cerebrospinal fluid; Glu, glucose; HHV-6, Human Herpesvirus 6; PCR, polymerase chain reaction; RBC, red blood cell; WBC, white blood cell. /, per.

HHV-6 DNA detected in the CSF, two had a positive result with fewer than 100 copies, and four had a positive result with a numerous of copies (cases 1, 2,3, and 4). Results and discussion will so focus on these four patients, their laboratory findings are summarized in -Table 1.

\section{Results}

\section{Case 1}

The first case is a 4-year-old boy who came at the emergency services for fever since 24 hours with headaches and alteration of its general condition. Clinical examination revealed a stiff neck without any other sign of infection. The child was lethargic but awake and oriented, he showed no sign of neurological focus. The brain computerized tomography (CT) scan was normal. Biological findings demonstrated an important inflammatory syndrome with pleocytosis for which a treatment with cefotaxime was started. Despite the treatment, clinical deterioration occurred 48 hours after his admission with emergence of a generalized pyramidal syndrome: tetraparesis with hyperreflexia and extended plantar reflexes. Intrathecal PCR of HHV-6 returned positive. Brain magnetic resonance imaging (MRI) was performed on the 3rd day and demonstrated T2-hyperintense lesion evoking an acute demyelinating encephalomyelitis (-Fig. 1). In this context, a treatment with intravenous immunoglobulin (Ig) was administered during 2 days but was not effective. At day 6 , a combination of high-dose corticosteroid therapy with ganciclovir for 3 days was administered. Subsequently, the child was progressively improved and his neurological examination returned to normal on the 13th day. A decrease of the MRI lesions was observed during the control performed 6 months later.

\section{Case 2}

The second case is a 3-year-old boy who had a left lateralized convulsion episode occurred during a first fever peak. The epileptic seizure stopped after 15 minutes, following the administration of intravenous diazepam. There were no signs of neurological or infectious focus, except headache. Biological assessment showed a mild inflammatory syndrome with pleocytosis. The brain CT scan was normal. The electroencephalogram demonstrated right posterior slowing. According to the institution's protocol, the child was hospitalized and treated with cefuroxime and acyclovir. Brain MRI, done on the day after admission, was normal. Cultures and herpes PCR returned negative at day 3, the antibiotherapy was stopped. Intrathecal PCR of HHV-6 was positive. There was no seizure recurrence.

\section{Case 3}

The third case is an 18-month-old boy who presented a generalized tonic-clonic status epilepticus during a first fever peak. In emergencies, the brain CT scan was normal, the biological assessment demonstrated a mild inflammatory syndrome and a normal cytorachia, and the electroencephalography (EEG) demonstrated widespread spike discharges developed from the left hemisphere. The seizure lasted 150 minutes and was controlled after intravenous administration of phenobarbital and continuous infusion of midazolam. Cerebral MRI realized 6 hours after the seizure onset showed T2-hypersignal and T1-hyposignal lesion focused on the corpus callosum. Antibiotic therapy with cefotaxime and acyclovir was given for 3 days, until herpes PCR and cultures returned negative. Intrathecal PCR of HHV6 was positive. Given the major febrile asthenia and the persistence of electrical discharge, intravenous ganciclovir was given for 7 days (from day 4 to day 11). Subsequently, the child's condition progressively improved without neurological sequelae highlighted. EEG returned to normal, and cerebral MRI performed 6 months later revealed a sequential cavitary lesion (-Fig. 2 ).

\section{Case 4}

The fourth case is a 2-year-old girl presented with brief but repeated lateral convulsions of her upper right limb in a febrile context. Clonic manifestations stopped after administration of intravenous midazolam, giving way to right 

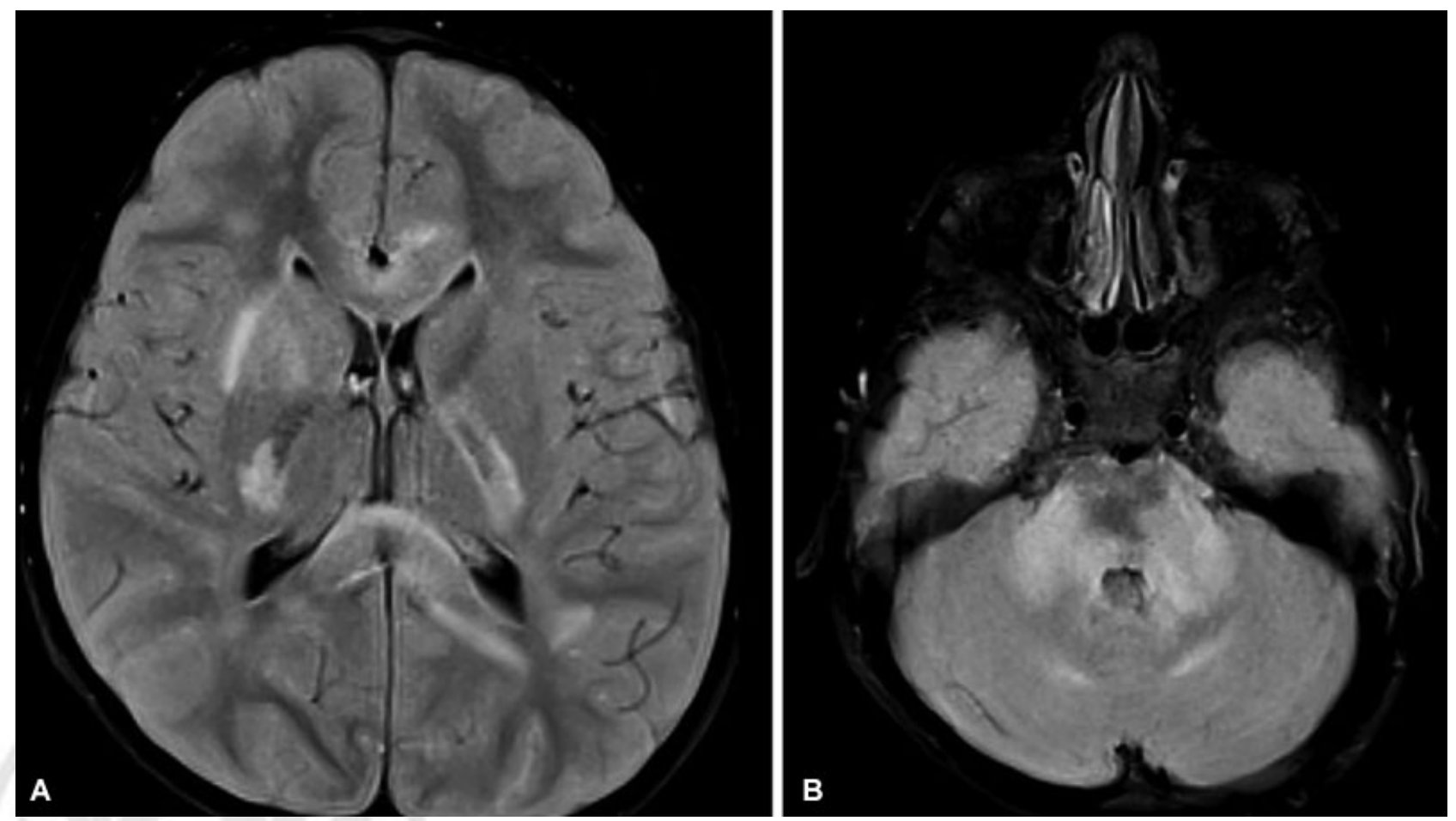

Fig. 1 Cerebral MRI of case 1 demonstrated T2/FLAIR hyperintense signals disseminated mainly in the bilateral internal capsule, the posterior corpus callosum, the left temporoparietal junction (A), and the brainstem (B). The location and appearance of the lesions corresponds to an acute disseminated encephalomyelitis (ADEM). FLAIR, fluid attenuation inversion recovery. MRI, magnetic resonance imaging.
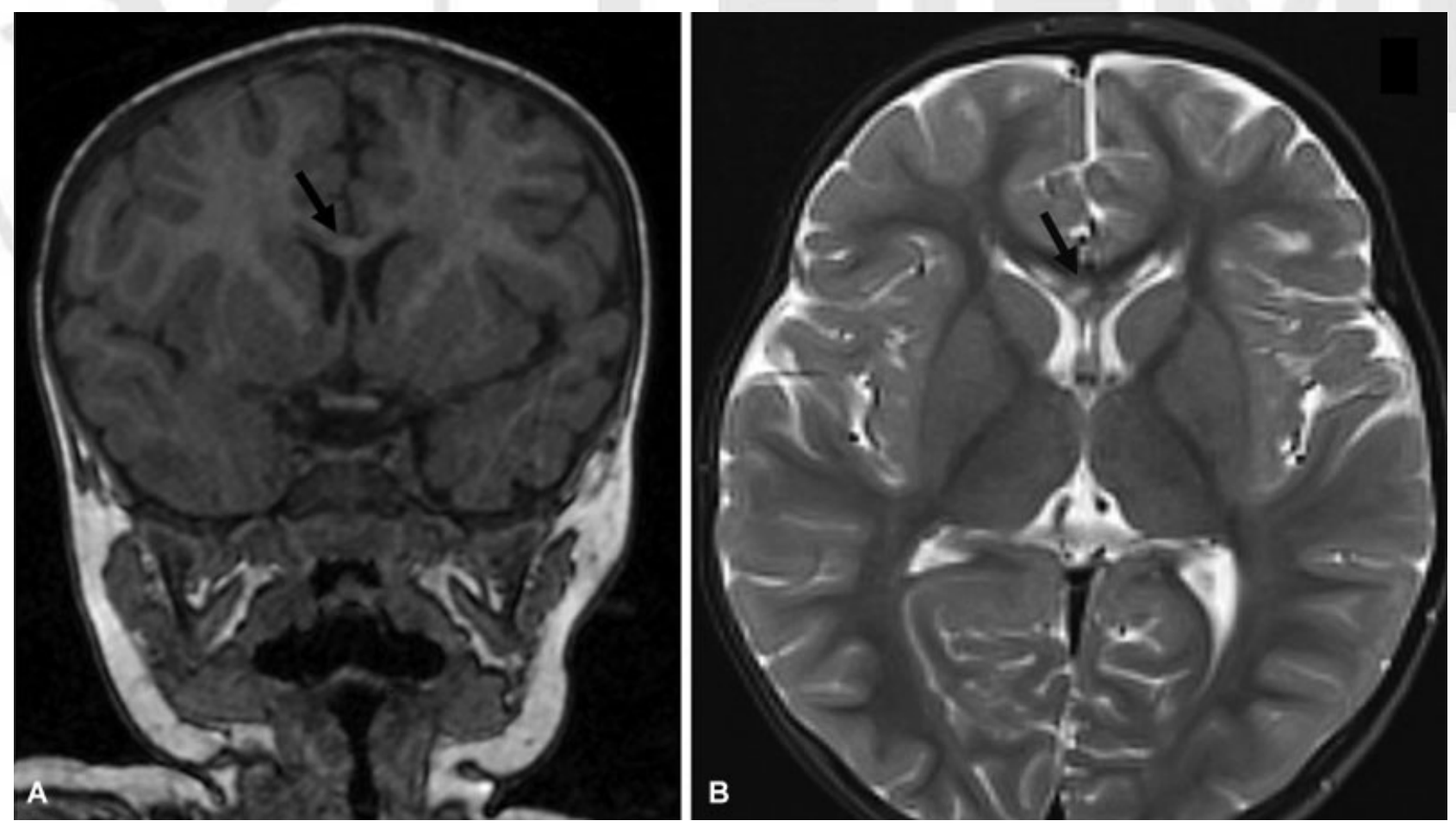

Fig. 2 Cerebral MRI of case 3 demonstrate a sequelar cavity (arrow) that appears normo/hyperintense in T1 (A) and hyperintense in T2 (B). MRI, magnetic resonance imaging.

hemiparesis which spontaneously resolved after a few hours. Biological findings and brain MRI were normal. EEG showed a postcritical slowing of the left hemisphere. Antiepileptic treatment with phenobarbital was initiated. Intrathecal PCR of HHV-6 returned positive.

\section{Discussion}

\section{Neurological Manifestations}

Primary HHV-6 infection may be accompanied by CNS disease. Clinical presentations are numerous and mainly 
include complicated febrile convulsions and encephalopathy. ${ }^{5}$ In immunocompromised patients, HHV-6 encephalopathy typically presents as acute limbic encephalitis with MRI changes in the hippocampus and amygdala. ${ }^{6}$ In addition to direct infection, HHV-6 has been proposed as a possible cofactor for the development of some demyelinating diseases, including acute disseminated encephalomyelitis. ${ }^{7}$ Consequently, it is logical to consider two possible mechanisms by which HHV-6 infection could cause neurological complications; direct invasion of the virus into the $\mathrm{CNS}^{4}$ and immune mediated CNS damage following HHV-6 infection. ${ }^{8}$

Finally, latent HHV-6 can sometimes reactivate and cause severe neurological complications in immunocompromised but also immunocompetent patients. ${ }^{4}$

\section{Investigations}

Serological response to a primary infection is characterized by a spike in IgM in the 1st week of illness, followed by a rise in IgG approximately 2 weeks later, which persists indefinitely. Anyway, several factors restrict the use of viral serology, including the delayed antibody response, the cross reactions between HHV-6A, HHV-6B, HHV-7, and cytomegalovirus (CMV) antibodies, the absence of a specific serological test is able to distinguish HHV-6A infections from HHV-6B infections, the absence of correlation between the absolute value or variations of the antibody titer and the presence of an active viral infection, and the alteration of the humoral immune response in some immunocompromised patients. Serological assays are indicated in only two cases, primary infection detected through seroconversion and through the identification of specific IgM and seroprevalence studies where seropositivity is indicative of a chronic latent infection.

An abnormal CSF profile is often but not always reported. ${ }^{9}$ Therefore, it would be imprudent to limit HHV-6 testing only to patients with abnormal CSF profiles.

Detecting genomic DNA by gene amplification (PCR) is currently the reference technique; it is accessible, specific, and very sensitive. Quantitative PCR methods, notably realtime PCR, constituted a significant advance as they reduced the risk of contamination by amplified DNA and provided a relatively precise value of the viral load in a given body organ or compartment. Detecting HHV-6 DNA in the CSF is highly indicative of an active infection of the CNS, especially of encephalopathy. This diagnosis must be considered even if the results of the concomitant DNAemia analysis are not significantly high (under a million copies of genomic DNA per milliliter of whole blood) as reactivation limited to the CNS may occur. However, since there have been reports of HHV-6 DNA detected in CSF specimens of immunocompetent individuals without CNS disease, ${ }^{10}$ it has been suggested that a positive PCR result should be interpreted on the basis of the clinical situation, after excluding others pathological agents. Nevertheless, a relatively high copy number (greater than $10^{3} / \mathrm{mL}$ ) of HHV-6 DNA in CSF measured through quantitative PCR strongly suggests HHV-6 active encephalitis. ${ }^{11}$.

So, in our cases, the presence of more than 1,000 copies of genomic DNA per milliliter in the CSF is highly indicative of an active infection. However, seroconversion and chromo- somal integration have not been considered, and there has been no control to assess the course of viremia.

\section{Treatment}

Clinical diagnosis of viral encephalitis before HHV-6 identification is usually suspected to be of herpes simplex virus (HSV) or varicella zoster virus (VZV) origin. Acyclovir therapy is administered but it has a very limited effect or no effect at all against roseoloviruses. ${ }^{12}$ When reactivation of HHV- 6 is recognized, anti-roseolovirus medication has to be initiated. Foscarnet and ganciclovir (valganciclovir) are the first line of defense and cidofovir is the second. Successful treatment has been published but randomized placebo-controlled trials are lacking. ${ }^{13}$ Given their potential side effects, whether antivirals are used depends on the patient's clinical situation.

As ganciclovir was proved to be effective and safe in the treatment of HHV-6 encephalitis, we chose to use this treatment in the two patients who present significant neurological involvement. A good therapeutic response has been observed without reported side effects.

\section{Outcome}

The outcome for HHV-6-positive patients is generally favorable, with most patients recovering fully. However, mild to moderate neurological impairment as well as death due to complications of neurological disease has been reported. ${ }^{14}$

\section{Conclusion}

HHV-6 has been demonstrated to be a neurotropic virus and has been linked to several neurological complications associated with a wide range of clinical outcomes. ${ }^{15}$

HHV-6 can invade the CNS in immunocompetent children. Therefore, the possibility of HHV-6 infection should be considered in children who present complex febrile seizure and acute encephalitis with white matter damages.

A combination of molecular detection and serology is ideal for study cases. Early detection of actively replicating HHV-6 in patients with clinical signs of encephalitis is important so that antiviral therapy can be initiated to attempt to resolve the neurological symptoms.

To our knowledge, ganciclovir treatment is safe and efficacious against severe neurologic disability. However, given the potentially severe nature of HHV-6 infections, we stress the need for developing and conducting therapeutic studies with a well-designed method to validate treatments for HHV-6 infections.

Conflict of Interest

None declared.

\section{References}

1 Yamanishi K, Okuno T, Shiraki K, et al. Identification of human herpesvirus- 6 as a causal agent for exanthem subitum. Lancet 1988;1(8,594):1065-1067

2 Komaroff AL, Boeckh M, Eliason E, Phan T, Kaufer BB. Summary of the $10^{\text {th }}$ international conference on Human Herpesviruses-6 and -7 (HHV-6A, -6B, and HHV-7). J Med Virol 2018;90(04):625-630 
3 Hall CB, Caserta MT, Schnabel KC, et al. Congenital infections with human herpesvirus 6 (HHV6) and human herpesvirus 7 (HHV7). J Pediatr 2004;145(04):472-477

4 Tavakoli NP, Nattanmai S, Hull R, et al. Detection and typing of human herpesvirus 6 by molecular methods in specimens from patients diagnosed with encephalitis or meningitis. J Clin Microbiol 2007;45(12):3972-3978

5 Ward KN, Andrews NJ, Verity CM, Miller E, Ross EM. Human herpesviruses- 6 and -7 each cause significant neurological morbidity in Britain and Ireland. Arch Dis Child 2005;90(06):619-623

6 Seeley WW, Marty FM, Holmes TM, et al. Post-transplant acute limbic encephalitis: clinical features and relationship to HHV6. Neurology 2007;69(02):156-165

7 De Bolle L, Naesens L, De Clercq E. Update on human herpesvirus 6 biology, clinical features, and therapy. Clin Microbiol Rev 2005;18 (01):217-245

8 Yoshikawa T, Asano Y, Akimoto S, et al. Latent infection of human herpesvirus 6 in astrocytoma cell line and alteration of cytokine synthesis. J Med Virol 2002;66(04):497-505

9 Friedman JE, Lyons MJ, Cu G, et al. The association of the human herpesvirus-6 and MS. Mult Scler 1999;5(05):355-362
10 Ward KN, Leong HN, Thiruchelvam AD, Atkinson CE, Clark DA. Human herpesvirus 6 DNA levels in cerebrospinal fluid due to primary infection differ from those due to chromosomal viral integration and have implications for diagnosis of encephalitis. J Clin Microbiol 2007;45(04):1298-1304

11 Kawamura Y, Sugata K, Ihira M, et al. Different characteristics of human herpesvirus 6 encephalitis between primary infection and viral reactivation. J Clin Virol 2011;51(01):12-19

12 Steiner I, Budka H, Chaudhuri A, et al. Viral meningoencephalitis: a review of diagnostic methods and guidelines for management. Eur J Neurol 2010;17(08):999-e57

13 Ongrádi J, Ablashi DV, Yoshikawa T, Stercz B, Ogata M. Roseolovirus-associated encephalitis in immunocompetent and immunocompromised individuals. J Neurovirol 2017;23(01):1-19

14 Olli-Lähdesmäki T, Haataja L, Parkkola R, Waris M, Bleyzac N, Ruuskanen O. High-dose ganciclovir in HHV-6 encephalitis of an immunocompetent child. Pediatr Neurol 2010;43(01): 53-56

15 Asano Y, Yoshikawa T, Kajita Y, et al. Fatal encephalitis/encephalopathy in primary human herpesvirus- 6 infection. Arch Dis Child 1992;67(12):1484-1485
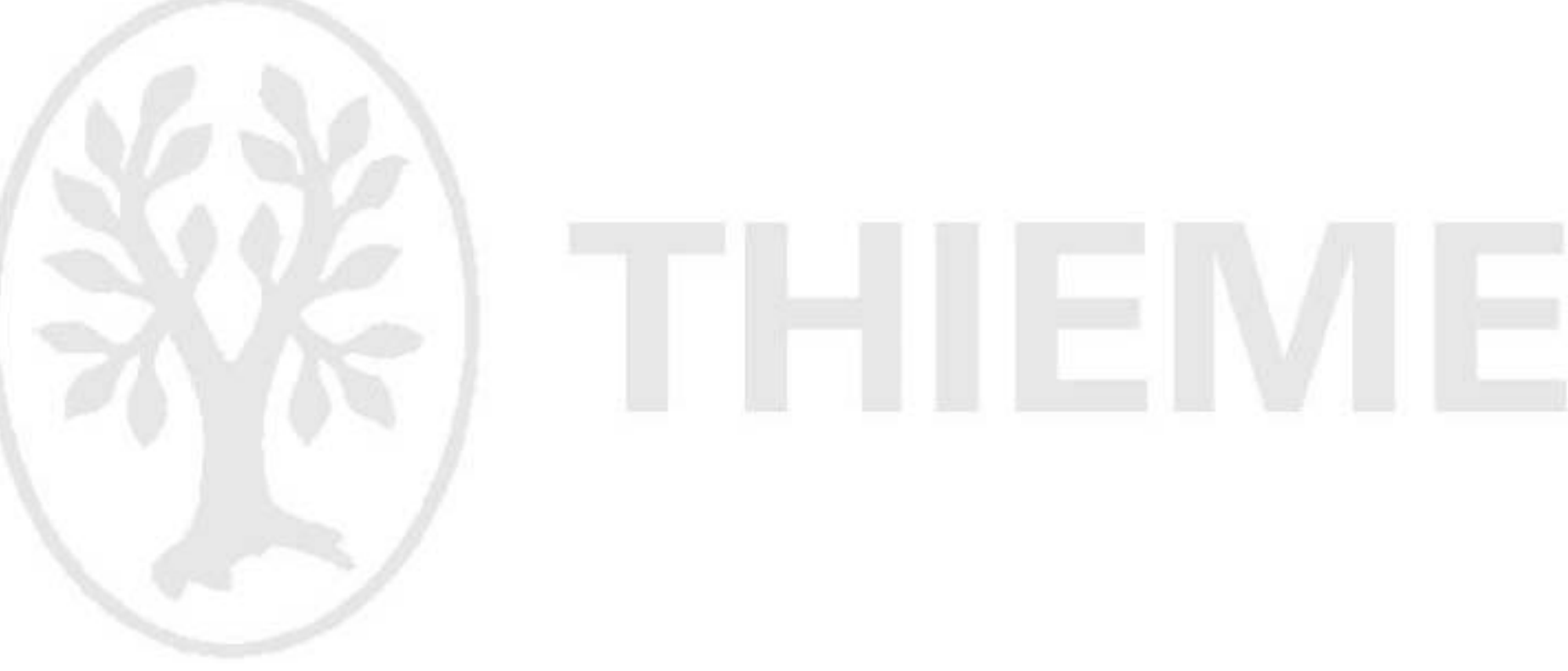